01

\title{
Инверсия вклада изотопа малой относительной концентрации в суммарный коэффициент поглощения смеси изотопов неона на переходе $3 s_{2}-2 p_{4}$
}

\author{
(C) Э.Г. Сапрыкин \\ Институт автоматики и электрометрии Сибирского отделения РАН, \\ 630090 Новосибирск, Россия \\ e-mail: Saprykin@iae.nsk.su
}

Поступила в редакцию 11.05.2018 г.

В окончательной редакции 02.04.2019 г.

Принята к публикации 09.04.2019 г.

При исследовании поглощения пробного лазерного излучения смесью четных изотопов неона в газоразрядной плазме методом магнитного сканирования перехода $3 s_{2}-2 p_{4}$ были разрешены с помощью численного моделирования контуры поглощения изотопов и обнаружено, что с уменьшением относительной концентрации одного из них его вклад в поглощение сменяется усилением. Выяснено, что эффект обусловлен радиационным механизмом передачи энергии возбуждения между атомами разного сорта при отсутствии различия энергий уровней (известном как „оптическая накачка“). Он оказался существенным для верхнего уровня перехода, переходы с которого в основное состояние разрешены, и отсутствует для нижнего уровня перехода, с которого такие переходы запрещены, но есть другие каналы распада.

Ключевые слова: оптическая накачка, изотопы неона, инверсия населенности, магнитное сканирование.

\section{Введение}

Основными причинами, порождающими когерентность вырожденных состояний, проявляющуюся при магнитном сканировании спектров, является анизотропия поля спонтанного излучения и поглощения излучения газоразрядной плазмой [1,2]. При этом много большая изотропная составляющая излучения индуцирует перенос и обмен магнитной (зеемановской) когерентностью [3]. Структура оптико-магнитных резонансов $(\mathrm{OMP})$ и нелинейная зависимость их ширин от давления (уширение и сужение) при обмене магнитной когерентностью, индуцированной собственным излучением разряда (СИЗ), была установлена в работе [4]. Вопросы индуцирования, переноса и обмена магнитной когерентностью уровней исследовались во многих экспериментальных работах, включая и опыты с использованием пробного (зондирующего) лазерного излучения, впервые реализованные в работе [5].

В свое время, исследуя специфику каскадного переноса когерентности в спектроскопии пробного поля, С.Г. Раутиан рассмотрел принципиальную возможность переноса оптической когерентности (поляризации) между уровнями, для которых разрешен дипольный переход [6]. Таким образом, к известным спонтанным радиационным процессам переноса населенности или магнитной когерентности от одного уровня к другому следует добавить третий тип - перенос поляризации (дипольного момента) от одного перехода к другому. Детально вопросы спонтанного переноса поляризации были рассмотрены в работе [7]. Было показано, что спонтанный перенос поляризации в дублете линий приводит к интерференционным изменениям спектра, схожим с нелинейным интерференционным эффектом (НИЭФ). В результате появляется асимметрия линий, расщепление и даже усиление в некоторых областях вместо поглощения.

Первая попытка обнаружения нового эффекта была предпринята авторами работы [8]. Для контроля формы спектра поглощения был выбран переход $3 s_{2}-2 p_{4}$ неона. Согласно [7], на нем эффект переноса поляризации не должен иметь место, и результаты измерений должны были служить для определения пороговой чувствительности экспериментальной установки к проявлению асимметрии спектральной линии. Для записи формы контура поглощения пробного поля был выбран метод магнитного сканирования как более простой и удобный с точки зрения контроля частотной отстройки полей относительно частот переходов. Если зондирующее излучение имеет круговую поляризацию, магнитное поле направлено параллельно распространению электромагнитной волны и $g$-факторы для нижнего и верхнего уровней одинаковы, как у перехода $3 s_{2}-2 p_{4}$ неона, то согласно сложившимся к тому времени представлениям магнитное сканирование полностью эквивалентно частотному.

Но оказалось, что это не так. В результате опыта обнаружили искажения формы допплеровского контура поглощения в виде его асимметрии при изменении частоты зондирующего излучения, которые не могли быть объяснены техническими или методическими причинами. Последующий теоретический анализ ситуации показал, что в данном опыте проявился нелинейный характер радиационных переходов, индуцированных СИЗ [9]. Это 
следствие расщепления зеемановских компонент $\sigma^{+}$и $\sigma^{-}$-излучения, распространяющихся поперек магнитного поля и имеющих одинаковые линейные поляризации. Поэтому в нуле магнитного поля интенсивности СИЗ этих компонент суммируются, а при расщеплении на ширину, большую допплеровской ширины, нет. При этом изменяются скорость вынужденного полем СИЗ переноса населенности уровней $1 s$ на уровень $2 p_{4}$ и поглощение зондирующего излучения на переходе $3 s_{2}-2 p_{4}$, что приводит к асимметрии формы линии при отстройке частоты лазерного излучения от центра зондируемого перехода. Таким образом, был обнаружен специфический эффект влияния СИЗ плазмы на ее оптические свойства (в данном случае на симметрию формы линии) - эффект самонасыщения оптических переходов.

В опытах [8] не проводилась численная обработка спектров $^{1}$, а используемые фабричные изотопы не были идеально чистыми ${ }^{2}$. Это послужило первой побудительной причиной повторить опыт [8], используя набор изотопических смесей изотопов при разных поляризационных условиях, разных частотах лазерного излучения и привлекая для численной обработки спектров модель, включающую эффект самонасыщения переходов. При этом выяснилось, что в условиях, когда реабсорбция излучения не мала и не слишком велика, поле СИЗ, порождаемое на концах протяженной кюветы, суммируется и достигает вследствие реабсорбции СИЗ максимума в ее центре, увеличивая коэффициент поглощения в однородном, как считалось ранее, положительном столбе тлеющего разряда [10]. Этим была подтверждена универсальность эффекта самонасыщения, проявляющегося и в отсутствие магнитного поля, в частности, в продольной неоднородности коэффициента поглощения в протяженных разрядах.

Но это не единственное новое явление, обнаруженное в опытах, поставленных для выяснения природы результатов [8]. Когда стали исследовать излучение разряда, пытаясь напрямую выявить прирост населенности вследствие эффекта самонасыщения, то были обнаружены необычные ОМР, смещенные относительно нуля магнитного поля. И это было удивительно, поскольку в опытах использовались четные изотопы неона без сверхтонкой структуры. Впоследствии было выяснено, что смещенные ОМР появляются при пересечении частот изотопически сдвинутых зеемановских компонент разных изотопов. Это свидетельствовало о наличии коллективных процессов синхронного излучения осцилляторами, принадлежащими пространственно разнесенным атомам. В недавней работе [11] были подведены промежуточные итоги экспериментального исследования таких резонансов, получивших название ОМР пересечения частот (ОМРПЧ), в излучении разряда.

\footnotetext{
1 Это было невозможно сделать ввиду отсутствия физической модели явления.

${ }^{2}$ В неоне-22 примесь изотопа неон-20 могла достигать $10 \%$.
}

Один из результатов работ состоял в том, что ОМРПЧ наблюдались на линиях ${ }^{20} \mathrm{Ne}$ с содержанием примесного изотопа ${ }^{22} \mathrm{Ne}$ всего $0.2 \%$, но с амплитудой некоторых резонансов, составляющей $\sim 1 \%$ от полной интенсивности излучения линии. Более того, при постепенном уменьшении относительной концентрации примесного изотопа с $\sim 8$ до $0.2 \%$ амплитуда ОМРПЧ, порождаемого переходом с уровня $2 s_{4}$ в основное состояние, возрастала, практически выходя на плато при концентрации примеси ниже $1.6 \%$ (рис. 8 в [11]). Это могло быть связано с тем, что населенность возбужденных уровней примесного изотопа намного превышает концентрацию, следующую из заселенности его нормального состояния вследствие обмена возбуждением с более сильно заселенными уровнями основного (буферного) изотопа. И это стало второй побудительной причиной к разрешению спектров магнитного сканирования смеси изотопов разных концентраций, которое, действительно, выявило относительный рост населенности уровня $3 s_{2}$ примесного изотопа при уменьшении его концентрации, приводящий к инверсии коэффициента поглощения на переходе $3 s_{2}-2 p_{4}$ (смене знака контура примесного изотопа при уменьшении его относительной концентрации).

\section{Эксперимент и методика обработки данных}

Настоящая работа, выполнена на оборудовании и по методике, которые использовались в работе [10]. Поэтому при описании опыта мы ограничимся повтором ключевых деталей, имея в виду возможность обращения к [10]. Тлеющий разряд возбуждался в капилляре диаметром $2.5 \mathrm{~mm}$ и длиной $40 \mathrm{~cm}$, помещенном в продольное магнитное поле и охлаждаемом водой. Измерялось поглощение смесей ${ }^{20} \mathrm{Ne}$ и ${ }^{22} \mathrm{Ne}$ с различными концентрациями изотопов. Для этого использовался стабилизированный $\mathrm{He}-\mathrm{Ne}$-лазер ЛГН-302 мощностью 1 мВт, работающий на изотопе ${ }^{20} \mathrm{Ne}$ и излучающий две ортогональные продольные линейно поляризованные моды с коэффициентом стабилизации лучше $10^{-8}$. Частота лазера стабилизировалась по равенству интенсивностей мод излучения, симметричных относительно центра линии. Разность частот излучения, зависящая от технологии, была указана как $640 \mathrm{MHz}$.

Схема двухпроходной установки, увеличивающей сигнал поглощения, представлена на рис. 1. Перед ячейкой находился поляризатор, выделяющий нужную ориентацию поляризации (переключающий частоту лазера), и фазовая пластинка, с помощью которой получались круговые поляризации разных знаков. Таким образом, для каждого из изотопов можно было реализовать 4 разные экспериментальные ситуации. Магнитное поле создавалось двумя соленоидами. Внутренний, более короткий модуляционный соленоид длиной $26 \mathrm{~cm}$ был расположен в области однородного магнитного поля сканирующего соленоида. Переменная часть магнитного 


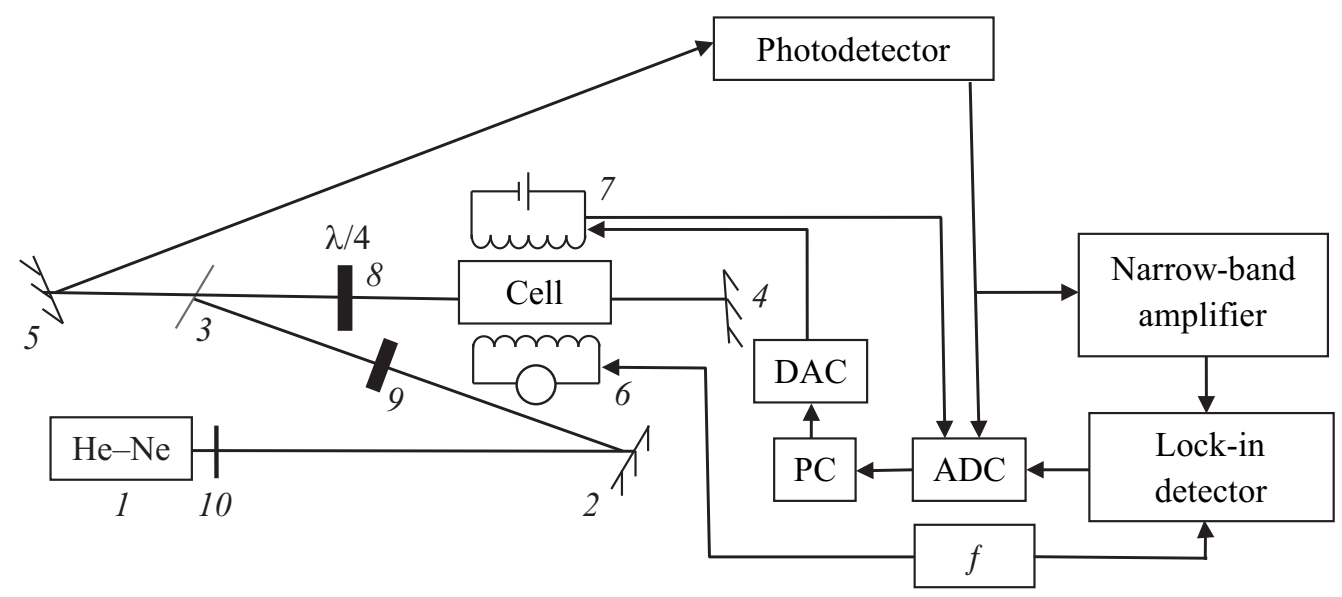

Рис. 1. Схема экспериментальной установки. 1 - стабилизированный лазер, $2-5$ - зеркала, 6 - модуляционный соленоид, 7 - сканирующий соленоид, 8 - фазовая пластинка $\lambda / 4,9-$ поляроид, $f-$ модуляционный генератор.

поля осциллировала с амплитудой 60 Ое. Напряженность магнитного поля в центре сканирующего соленоида длиной $32 \mathrm{~cm}$ вычислялась по данным АЦП, пропорциональным току соленоида. Ранее при исследовании эмиссионных ОМРПЧ (см. [11] и ссылки в ней) использовался 12-разрядный АЦП и калибровка шкалы магнитного поля была „привязана“ к максимальному значению неоднородного магнитного поля в центре соленоида, что составляло 1.16 Ое на квант АЦП-12. При переходе к исследованию ОМРПЧ в поглощении $[10,12]$ при той же схеме измерения тока стал использоваться 16-разрядный АЦП. Для сохранения преемственности с опытами предыдущих лет была оставлена неизменной прежняя калибровка магнитной шкалы, составившая при пересчете к кванту АЦП-16 в 8 раз меньшую величину 0.145 Oe/quantum. Именно в таком масштабе отображены шкалы осей абсцисс на графиках [10,12] и данной работы.

Для повышения точности отсчета магнитных полей в программу обработки данных был введен множитель $s m$, позволяющий корректировать коэффициент пересчета квантов АЦП в напряженность магнитного поля при наличии более точных реперов шкалы. В данном случае таким репером был изотопический сдвиг (ИС) перехода $3 s_{2}-2 p_{4}$, равный $907.1 \pm 2.5 \mathrm{MHz}$ (в единицах напряженности магнитного поля $497 \mathrm{Oe})$, полученный нами по результатам усреднения известных в литературе ИС уровней данного перехода. Пространственная неоднородность магнитного поля данного соленоида (характер уменьшения поля на концах) была описана в [13]. В случае исследования поглощения использование результатов [13] приводит к усредненному коэффициенту пересчета квантов в эрстеды, равному 0.138 Oe/quantum, a не 0.145 Oe/quantum, как для центра соленоида, что должно давать $s m=0.95$.

После прохождения ячейки с газом луч попадал на кремниевый фотодетектор. Далее сигнал поступал в канал, регистрирующий постоянную составляющую, и в канал, регистрирующий модуляционную составляющую. В нем сигнал фотодетектора усиливался на частоте осцилляций магнитного поля узкополосным усилителем, и синхронно детектировался. После этого сигналы оцифровывались АЦП и передавались на компьютер. При этом усредняемые по отдельности кривые прямого и обратного проходов визуализировались на экране монитора в реальном времени, а после завершения сканирования документировались в виде файлов прямого и обратного проходов для последующего анализа.

\section{Моделирование кривых}

Параметры зарегистрированной кривой оценивались по методу наименьших квадратов (МНК) в предположении, что линии поглощения изотопов имеют допплеровскую форму контура и изотопически смещены. Асимметрия контуров поглощения, являющаяся следствием насыщения оптических переходов полем СИЗ разрядной кюветы в присутствии магнитного поля, учитывалась добавкой в модель контуров специфической формы $(F S S)$ [10]. При этом необходимо учитывать реабсорбцию поля СИЗ. Вопросы деформации формы линий газоразрядных источников света при реабсорбции излучения хорошо освещены в литературе. Специфика рассматриваемого случая самонасыщения в том, что в нем важны эффекты изменения формы линий излучения не с торца излучателя, а внутри его объема. Эти вопросы детально рассмотрены в [10], где для описания формы линии эффекта самонасыщения была сконструирована функция

$$
\begin{aligned}
& F S S(x)=\exp \left(-\left(\frac{x}{k v_{s s}}\right)^{2}\right) \\
& +k_{s s} \exp \left(-\left(\frac{1.17 k v_{s s}-|x|}{0.55 k v_{s s}}\right)^{2}\right) .
\end{aligned}
$$




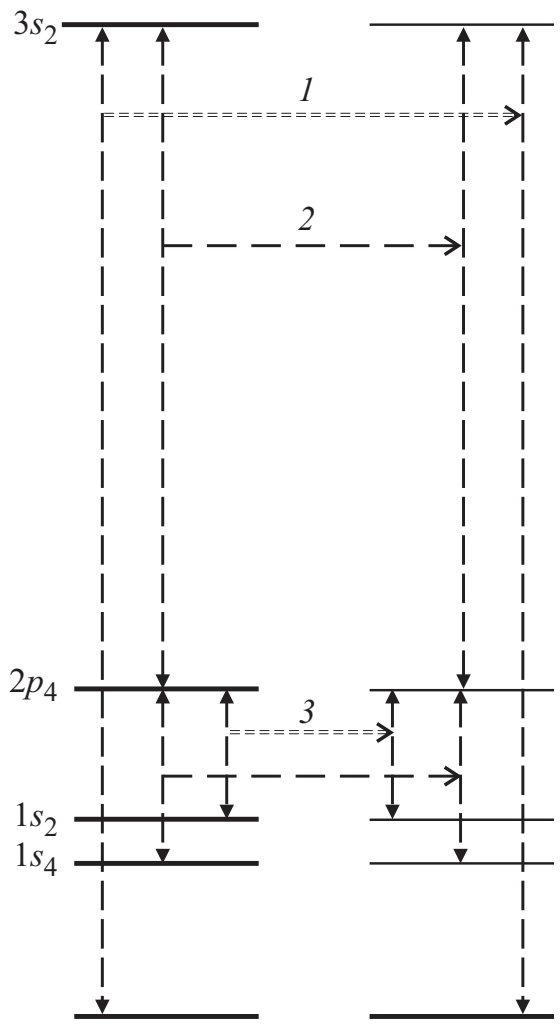

Рис. 2. Схема термов изотопов неона, демонстрирующая радиационный перенос населенности между уровнями изотопов: 1 - оптическая накачка, 2, 3 - самонасыщение.

За форму функции (1) ответственны параметры $k_{s s}$ и $k v_{s s}$. При $k_{s s}=0$ форма контура гауссова, а при $k_{s s}=0.174$ близка к треугольной. В разряде неона прирост населенности уровня $2 p_{4}$ определяется действием СИЗ нескольких переходов, сильно различающихся и силами осцилляторов, и населенностями уровней. Это переходы $2 p_{4} \rightarrow 1 s_{2}, 1 s_{4}, 1 s_{5}$ и переходы $1 s_{2}, 1 s_{4} \rightarrow^{1} S_{0}$ (рис. 2), где ${ }^{1} S_{0}-$ основное состояние, в обозначениях Пашена отсутствующее, причем для последних переходов возможно полное пленение излучения на диаметре разрядной трубки, и форма суммарного контура самонасыщения сложная. В виду этого при обработке результатов мы включили в программу два контура вида (1) с амплитудами $A_{s s}$ и $A_{s s 2}$, т. е. описывали эффект самонасыщения шестью параметрами.

Добавлялись и другие функции, моделирующие подкладку допплеровских контуров изотопов. В итоге контур поглощения смеси изотопов описывался с помощью функции:

$$
\begin{aligned}
I(x) & =\left(1+A_{s s} F S S(x)\right)\left(1+A_{s s 2} F S S_{2}(x)\right) \\
& \times(1+\operatorname{AgmFGM}(x))\left(A_{22} F O M(x)+A_{20} F D I(x)\right),
\end{aligned}
$$

где $x=s m H-$ корректированные значения магнитного поля сканируемого соленоида; $A_{s s}$ и $A_{s s 2}$ - амплитуды двух контуров, включенных в программу для описания эффекта самонасыщения; параметры $A_{22}$ и $A_{20}$ - амплитуды смещенных контуров ${ }^{22} \mathrm{Ne}(F D I)$ и ${ }^{20} \mathrm{Ne}(F O M)$. Амплитуды $A_{22}$ и $A_{20}$ измерялись в единицах концентрации изотопов. При этом концентрация буферного изотопа вводилась как известная величина, а концентрация примесного изотопа оценивалась по результатам опытов. Для описания величины сигнала в каждом опыте вводились амплитуды $W$, на которые умножался контур $I(x)$. Формы контуров ${ }^{20} \mathrm{Ne},{ }^{22} \mathrm{Ne}$ и гальваномагнитного контура $(F G M)$ имели следующий вид. Контур ${ }^{20} \mathrm{Ne}$ :

$$
\begin{aligned}
F O M(x) & =(1-a s) \exp \left(-\left(\frac{O M-s x}{k v_{20}}\right)^{2}\right) \\
& +a s \exp \left(-\left(\frac{O M+s x}{k v_{20}}\right)^{2}\right) .
\end{aligned}
$$

Контур ${ }^{22} \mathrm{Ne}$ :

$$
\begin{aligned}
F D I(x) & =(1-a s) \exp \left(-\left(\frac{O M-D I-s x}{k \nu_{22}}\right)^{2}\right) \\
& +a s \exp \left(-\left(\frac{O M-D I+s x}{k v_{22}}\right)^{2}\right) .
\end{aligned}
$$

Гальваномагнитный контур, центрированный в нуле магнитного поля:

$$
F G M(x)=1-\exp \left(-\left(\frac{x}{k v_{g m}}\right)^{2}\right) .
$$

Здесь $s= \pm 1-$ знак круговой поляризации, $D I-$ изотопический сдвиг линии, $O M-$ отстройка частоты лазера от частоты перехода ${ }^{20} \mathrm{Ne}$ в разрядной кювете, $k v_{20}, k v_{22}$ - допплеровские параметры ширин линий ${ }^{20} \mathrm{Ne}$ и ${ }^{22} \mathrm{Ne}$, выраженные в единицах напряженности магнитного поля $(\mathrm{Oe})$. Для сокращения числа варьируемых параметров $k v_{20}$ вычислялся исходя из различия масс изотопов как $1.0488 k v_{22}$. Неточности установки круговой поляризации (наличие примеси линейной поляризации) учитывались множителем as в (3) и (4).

Положение нуля шкалы ординат задавалось параметром $x_{0}\left(x \rightarrow x-x_{0}\right)$. Сдвиг нуля напряжения синхронного детектора задавался параметром $y_{0}$. Наклон нулевой линии из-за возможного влияния магнитного поля на элементы оптической схемы учитывался параметром $b_{0}$. Данные параметры вводились на этапе вычисления производной сигнала как разности двух значений функции (2). Окончательная аппроксимирующая функция имела вид

$$
\begin{aligned}
\frac{d I(x)}{d x} & =k_{w} W\left[I\left(x-x_{0}+\varepsilon\right)-I\left(x-x_{0}-\varepsilon\right)\right] \\
& +y_{0}+b_{0}\left(x-x_{0}\right) .
\end{aligned}
$$

Здесь $\varepsilon-$ константа, описывающая амплитуду гармонической модуляции магнитного поля $H$ (девиацию), $k_{w}-$ дополнительная нормирующая константа. 
Для моделирования каждого из опытов требуются 20 параметров [10]. Поскольку время оценки параметров в МНК пропорционально квадрату числа узлов аппроксимации (точек кривой), то на первом этапе подготовки данных проводилось усреднение данных прямого и обратного проходов в одну кривую. Это в 4 раза уменьшало время счета сравнительно с аппроксимацией двух кривых без уменьшения точности решения обратной задачи, поскольку при усреднении данных двух однотипных опытов в 2 раза уменьшалась и дисперсия ошибок суммарной кривой.

Время решения обратной задачи МНК пропорционально третьей степени числа оцениваемых параметров (у нас таких 17 на одну кривую). Поэтому если оценивать параметры всех 8 кривых, то время счета будет чрезмерным. Для уменьшения числа варьируемых параметров, повышения точности оценки и уменьшения времени счета применялась совместная обработка всего семейства кривых. При этом сокращение количества оцениваемых параметров обеспечивается введением параметров, общих для всего семейства. Действительно, индивидуальных для каждой кривой параметров всего 5 $\left(W, s, A_{20}, A_{22}, O M\right)$, причем только 3 из них нуждаются в оценке, а 2 ( $s$ и концентрация буферного изотопа) константы. Остальные параметры можно обобщить, добавляя на каждую новую кривую всего по 3 варьируемых параметра. Параметр $D I$ перехода $3 s_{2}-2 p_{4}$ вводился как константа, равная 497 Ое.

\section{Нормировка графиков в опытах с поглощением лазерного излучения}

Специфика метода поглощения состоит в том, что сигнал определяется поглощением пробного поля в исследуемой смеси газов. Поэтому чем больше поглощение, тем больше сигнал. В силу резонансного характера поглощения при сканировании магнитного поля меняется интенсивность лазерного излучения на выходе из среды. В наших опытах ослабление интенсивности излучения достигало $15 \%$. В результате выражение (6), записанное в приближении постоянства интенсивности зондирующего излучения, становилось неверным. Поэтому перед счетом осуществлялось нормирование сигнала в канале, регистрирующем модуляционную составляющую, на интегральный сигнал с фотодетектора. Сначала осуществлялась нормировка данных интегрального сигнала на максимум интенсивности лазера в отсутствие поглощения. При этом за максимум принималась интенсивность сигнала на краю диапазона сканирования магнитного поля, где поглощение было минимальным. В итоге нормированный интегральный сигнал отображался как провал в интенсивности - от единичного значения в крыльях до минимума в центре, зависящего от поглощения. Затем сигнал производной в таблице данных опыта делился на нормированный прямой сигнал, восстанавливая контур, отвечающий тонкому слою.

\section{Результаты измерений}

В опытах использовались обогащенные смеси фабричных изотопов неона - одна с содержанием ${ }^{20} \mathrm{Ne} 99.8 \%$, а другая - с содержанием ${ }^{22} \mathrm{Ne} 92.2 \%$. Представлены результаты для 6 порций ${ }^{20} \mathrm{Ne}(p=0.72$ Torr $)$ и для 6 порций ${ }^{22} \mathrm{Ne}(p=0.84$ Torr). Проведено 8 опытов с такими смесями при токе $20 \mathrm{~mA}$, отображенных на рис. 3 . Символы точек сигнала производной - это данные опыта, корректированные с помощью описанной выше нормировки данных. Сплошные линии демонстрируют результаты МНК-подгонки параметров модели. Номера кривых обозначают их порядковый номер в семействе обрабатываемых кривых, знаки $(+,-)$ знаки круговой поляризации лазерного излучения $s$. Графики $1-4$ относятся к ${ }^{20} \mathrm{Ne}$, а $5-8$ к ${ }^{22} \mathrm{Ne}$. Каждая из представленных кривых зависит от 20 параметров, причем 17 из них нуждаются в оценке из данных опыта. Семейство из 8 кривых описывают 62 параметра, причем 44 из них оцениваемые параметры. В данном тексте мы ограничимся представлением и анализом лишь основных из них.

Бросается в глаза асимметрия сдвигов кривых одной поляризации в ${ }^{20} \mathrm{Ne}$ относительно нуля оси абсцисс, отвечающих разным частотам $(1+, 3+$ и $2-, 4-)$, хотя и в лазере, и в ячейке используется ${ }^{20} \mathrm{Ne}$, и сдвиги должны быть симметричны. Это связано с тем, что линия усиления гелий-неонового лазера, по контуру которой стабилизируется его частота, смещена относительно линии поглощения чистого ${ }^{20} \mathrm{Ne}$ в кювете вследствие разного сдвига линий неона при столкновениях с гелием и неоном. При этом для одинаковых круговых поляризаций разных частот должен быть одинаковым параметр $\Sigma$, отвечающий разности абсолютных значений смещения контуров относительно нуля оси абсцисс (условие $\Sigma=J M+O M 3=O M 4+O M 2=O M 8+O M 5=$ $=O M 7+O M 6)$. Однако численная аппроксимация экспериментальных кривых показала, что в случае обобщения параметра $a_{s}$ и использования его средней величины точность подгонки ухудшается $\left(\chi^{2}\right.$ растет) сравнительно с индивидуализацией оценок для кривых семейства. Физически это может быть обусловлено погрешностями перенастройки оптической схемы на новые поляризационные условия опыта или временным дрейфом параметров установки. В нашем случае условие равенства асимметрии смещений контуров реализовывалось за счет индивидуализации оценок параметра $a_{s}$ в диапазоне значений от -0.022 до +0.031 при среднем значении -0.002 .

Как известно, при столкновениях с атомами гелия линия $3 s_{2}-2 p_{4}$ неона испытывает сдвиг в синюю область спектра со скоростью $21 \pm 3 \mathrm{MHz} /$ Torr [14]. Что касается сдвига линии неона из-за столкновений с атомами собственного газа, то в литературных данных второй половины 20-го века долгое время не было согласия не только по величине, но и по знаку сдвига. Таких работ было много, измеренный сдвиг изменялся от $1 \mathrm{MHz} /$ Torr 


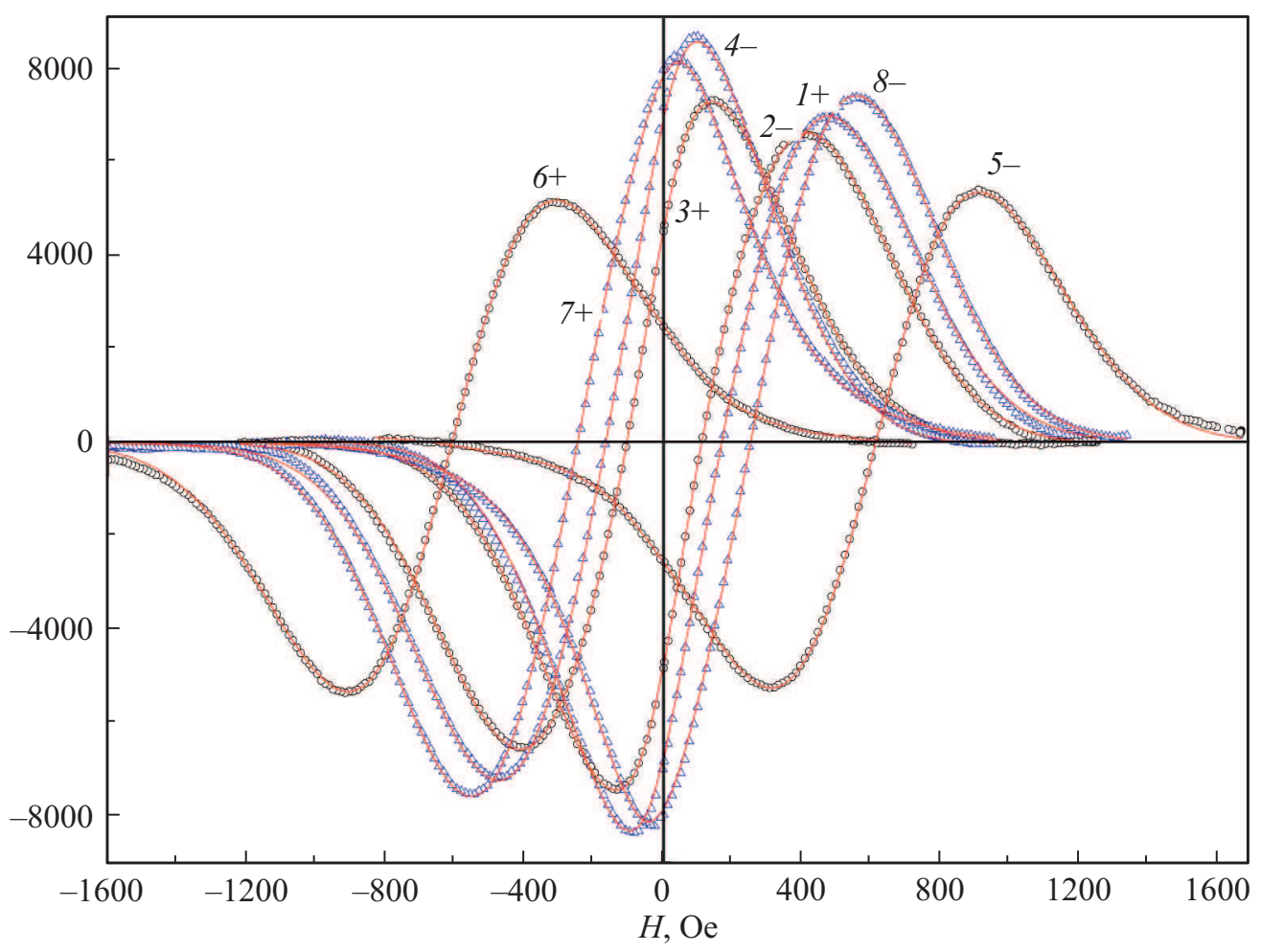

Рис. 3. Модуляционная составляющая сигнала в обогащенных изотопах. Кривые с номерами $1-4$ относятся к ${ }^{20} \mathrm{Ne}$, а с номерами $5-8$ к ${ }^{22} \mathrm{Ne}$. Символы у графиков - порядковые номера в семействе обрабатываемых кривых и знаки круговой поляризации. Графики $1+, 4-, 7+, 8-$ отвечают более высокой частоте лазера, а графики $2-, 3+, 5-, 6+-$ более низкой частоте.

(красный сдвиг) до $-25 \mathrm{MHz} /$ Torr (синий сдвиг), при этом в совокупности со столкновительным уширением каждый такой опыт удовлетворял теории ван-дерваальсова взаимодействия. Только в работе [15] было обращено внимание на то, что в опытах определялся суммарный сдвиг, обусловленный столкновениями как с атомами, так и с электронами. Из ее результатов, полученных при $300 \mathrm{~K}$, следовало, что сдвиг описывается выражением

$$
\begin{aligned}
\Delta_{\mathrm{cr}} & =\Delta_{a}+\Delta_{i}=(6.6 \pm 1.5) \mathrm{MHz} / \operatorname{Torr} /\left(\mathrm{A} / \mathrm{cm}^{2}\right) \\
& -(7.6 \pm 0.7)(\mathrm{MHz} / \text { Torr }) /\left(\mathrm{A} / \mathrm{cm}^{2}\right) .
\end{aligned}
$$

Конкретно в кювете диаметром $2.4 \mathrm{~mm}$, использованной в [15], красный атомный сдвиг $\Delta a=6.6 \mathrm{MHz} /$ Torr с ростом тока разряда линейно уменьшался до нуля при токе $40 \mathrm{~mA}$, а затем изменял знак, и при токах до $150 \mathrm{~mA}$ линейно рос синий электронный сдвиг $\Delta_{i}{ }^{3}$. Поэтому в ячейке с неоном при токе $20 \mathrm{~mA}$ и давлении 0.8 Torr следует ожидать красный сдвиг $\sim 2.5 \mathrm{MHz}$. Для данного лазера нам не известно точно давление гелия в активном элементе, однако в коротких и узких капиллярах, используемых в таких лазерах, оно должно

\footnotetext{
3 Работа [15] прекратила поток разноречивых публикаций об уширении данной линии неона за счет ван-дер-ваальсова взаимодействия, подтвердив теорию Казанцева [16], согласно которой уширение линии $6328 \AA$ атомными столкновениями определяется перекрытием электронных оболочек атомов.
}

быть около 5 Torr. Для давления гелия 5 Torr получаем сдвиг частоты $105 \pm 15 \mathrm{MHz}$. В наших опытах получено значение $63 \pm 10 \mathrm{Oe}$, т.е. $115 \pm 18 \mathrm{MHz}$, что близко к ожидаемому сдвигу.

На параметр $\Delta$, отвечающий сумме абсолютных значений смещения контуров относительно нуля оси абсцисс, налагалось условие $\Delta=O M-O M 3=O M 4-O M 2=$ $=O M 8-O M 5=O M 7-O M 6$, обусловленное частотным разделением мод лазера. Каждая из этих разностей отвечает одинаковой круговой поляризации в ячейке, но разным линейным поляризациям (частотам), из которых она формируется. В наших опытах было получено значение $345.4 \mathrm{Oe}(630.5 \mathrm{MHz})$, близкое к $\sim 640 \mathrm{MHz}$, указанной в документации лазера.

Допплеровский параметр был оценен как $404 \pm 14$ Ое, что близко к оценке $412 \mathrm{Oe}$, расчетной для температуры $300 \mathrm{~K}$. Амплитудные множители $W$, характеризующие уровень сигнала (6) в каждом из 8 опытов, отрицательны, что отвечает поглощению излучения. Знаки амплитуд контуров самонасыщения $A_{s s}$ и $A_{s s 2}$ получились положительными, что с учетом отрицательного знака параметра $W$ отвечает росту поглощения, т.е. населенности уровня $2 p_{4}$. В сумме эти два контура, как и в [10], описывают структуру, весьма похожую на производную сильно реабсорбированных линий излучения. Эта структура индуцируется напрямую совместным излучением трех переходов с уровней $2 p_{4}$ на $1 s$-уровни 
и опосредованно излучением еще двух переходов с резонансных уровней в основное состояние, вносящих вклад в населенность уровней $1 s_{2,4}$. Эти переходы отличаются вероятностями, населенностью нижних уровней, степенью вырождения, факторами Ланде. Извлечь из этих обобщенных результатов детальную физическую информацию невозможно. Равным образом из полученных данных нельзя сделать заключение о характере гальваномагнитного эффекта (параметры $A_{g m}, k v_{g m}$ ). Здесь ошибки определения параметров на порядок величины превышали их значения.

При аппроксимации кривых определялась усредненная по семейству кривых относительная концентрация примесного изотопа ${ }^{22} \mathrm{Ne}$ в ${ }^{20} \mathrm{Ne}$ (условие $\left.A_{22}=A_{22} \_2=A_{22} \_3=A_{22} \_4\right)$ и ${ }^{20} \mathrm{Ne}$ в ${ }^{22} \mathrm{Ne}$ (условие $\left.A_{20 \_5}=A_{20} 6=A_{20} \_7=A_{20} 8\right)$. Эти ограничения обусловлены постоянством соотношения концентраций изотопов в этих смесях. Относительная амплитуда примесного контура $A_{22}$ при содержании изотопа ${ }^{20} \mathrm{Ne}$, равном $99.8 \%$, определилась как $-1.86 \%$ (т. е. отвечала просветлению, а не поглощению), а для контура $A_{20}$ при концентрации изотопа ${ }^{22} \mathrm{Ne}$, равной $92.2 \%$, как $+3.18 \%$ (т.е. отвечала поглощению, но для заметно меньшей концентрации примеси). При этом погрешности оценок амплитуд разных кривых менялись от 0.25 до $1.6 \%$.

Для параметра $s m$ получено значение $0.94 \pm 0.045$, которое заметно отличается от представленного ранее в работах $[10,12]$. Так, в [10] для смеси изотопов равных концентраций было получено значение $1.01 \pm 0.02$, отвечающее значению магнитного поля в центре соленоида сканирования. В условиях неоднородного магнитного поля соленоида сканирования данной установки [13] это связывалось с продольной неоднородностью коэффициента поглощения в смеси изотопов неона, обусловленной насыщающим действием поля СИЗ разряда, увеличивающим населенность уровня $2 p_{4}$ в центре разрядной трубки [10]. В работе [12], где измерялся ИС нижних резонансных переходов $1 s_{2,4}-{ }^{1} S_{0}$, значение $s m=1.01$ обеспечило совпадение результатов с независимыми результатами работы [17], что можно рассматривать как дополнительное подтверждение этого значения $s m$. Там же для высоковозбужденных ИК переходов с малым СИЗ получился результат, близкий к ожидаемому для усредненного магнитного поля соленоида $0.138 \mathrm{Oe} / q u a n t u m$ и отвечающий значению $s m=0.95$.

Для проверки достоверности инверсии коэффициента поглощения следует выяснить - насколько изменение масштаба шкалы абсцисс (параметра $s m$ ) может сказаться на смещениях и амплитудах контуров, отвечающих примесным изотопам различной концентрации. С этой целью в дополнительных численных опытах параметр sm принимался за константу, меняющуюся от 0.94 до 1, и анализировалось изменение ключевых параметров. Параметр $A_{22}$ сохранял при этом отрицательный знак, меняясь от -1.86 до $-2.1 \%$, а параметр $A_{20}$ варьировался в области от 3.17 до 3.24\%. Параметр ширины

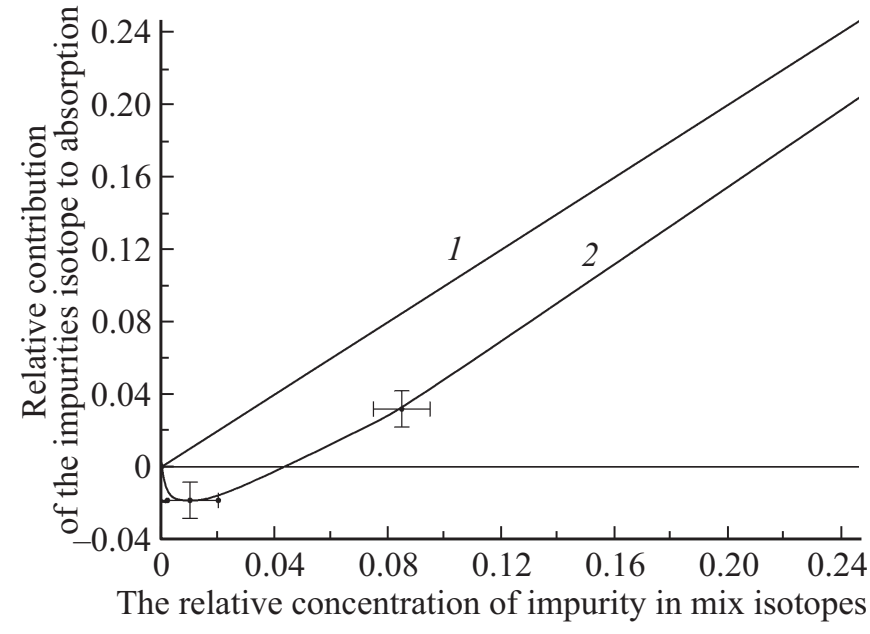

Pис. 4. Демонстрация инверсии коэффициента поглощения примесного изотопа при уменьшении его относительной концентрации. График 1 отвечает отсутствию обмена населенностью между уровнями изотопов, график 2 - реальный вклад примесного изотопа в поглощение излучения.

линии $k v_{22}$ изменялся от 404 до $418 \mathrm{Oе}$, расщепление частот линейных поляризаций лазера $\Delta$ определялось как 630-633 MHz, а разность частот перехода $3 s_{2}-2 p_{4}$ неона в исследуемой кювете и активном элементе лазера имела разброс от 109 до $115 \mathrm{MHz}$. В частности, при значении $s m=1$ были получены следующие значения ключевых параметров: $A_{22}=-2.2 \%, A_{20}=+2.94 \%$, $k v_{22}=417.6 \mathrm{Oe}, \Delta=632.8 \mathrm{MHz}, \Sigma=109 \mathrm{MHz}$. Таким образом, вариации параметра $s m$ мало влияют на ключевые результаты опыта, и презентуемая данной работой инверсия коэффициента поглощения примесного изотопа проявляется с достоверностью, не зависящей от величины $s m$.

Возможные причины уменьшения $s m$ в данных опытах заключаются в том, что в настоящей работе парциальное давление основного изотопа было существенно большим (0.8 Torr), чем в [10,12] (4 и 0.13 Torr). Это увеличивает пленение излучения и уменьшает продольную пространственную неоднородность поглощения, обусловленную реабсорбцией излучения и самонасыщением оптических переходов.

График зависимости вклада примесного изотопа в поглощение от его концентрации в исходной смеси представлен на рис. 4. На нем приведен участок кривой, отвечающий концентрациям примесного изотопа, меньшим 20\%. График характеризуется большими погрешностями отношения исходных концентраций изотопов, отложенных по оси абсцисс. Это связано с тем, что при наполнении кюветы разными изотопами они сорбировались пористым катодом с последующей десорбцией в разряд, и определение реальных концентраций изотопов в положительном столбе является непростой задачей. Особенно это относится к ${ }^{20} \mathrm{Ne}$, где исходное содержание примеси ${ }^{22} \mathrm{Ne}$ менее $0.2 \%$ при последующем напуске 
в кювету без многоразового отжига разрядом в гелии с сопутствующей откачкой могло повышаться на порядок.

На опыте хорошо определяются точки графика, отвечающие равной смеси изотопов, где оптической накачки нет и контуры изотопов проявляются с одинаковой амплитудой (точка $X=Y=1$ ), и отсутствию примесного изотопа (точка $X=Y=0$ ). Прямая линия 1 , соединяющая эти точки, отвечает отсутствию обмена населенностью уровней изотопов. Бета-сплайн 2 моделирует изменение вклада примесного изотопа в поглощение при уменьшении его относительной концентрации в газе. Опыт показал, что амплитуда контура примесного изотопа уменьшается быстрее, чем его концентрация в смеси изотопов. Так, уже при концентрации примеси около $4 \%$ населенность уровня $3 s_{2}$ примесного изотопа сравнивается с населенностью уровня $2 p_{4}$, и контур примесного изотопа исчезает. При дальнейшем уменьшении концентрации контур меняет знак, уменьшая суммарное поглощение смеси изотопов на магнитных полях, отвечающих положению примесного изотопа (по абсолютной величине достигая $2 \%$ концентрации примеси). При стремлении содержания примеси к нулю инверсия может еще возрасти, но потом она, конечно, должна исчезнуть вместе с уменьшением до нуля концентрации примесного изотопа ${ }^{22} \mathrm{Ne}$. Однако для уточнения формы графика в этой области необходимо увеличить число точек измерения при разных соотношениях концентраций изотопов с повышением точности подготовки исходных смесей, что в наших условиях было невозможно сделать. Ввиду отсутствия определенности с реальным содержанием примеси ${ }^{22} \mathrm{Ne}$ в кювете при малых концентрациях и для обеспечения плавного перехода сплайна в ноль мы отнесли результат измерения при минимальной концентрации $(-2 \%)$ к трем точками концентрации примеси $(0.2,1$ и $2 \%)$.

Для визуализации результатов численного анализа опытов, приведем на рис. 5 кривые двух опытов $6+$ и $3+$ из 8 , отображенных на рис. 3 . В них из модели обработки после подгонки были исключены контуры, описывающие примесные изотопы, и отображалось увеличенное в 10 раз различие между экспериментальной кривой и кривой, описываемой такой укороченной моделью. График 6+ это опыт с изотопом неон-22, в котором высокое содержание примеси неона-20. При удалении ${ }^{20} \mathrm{Ne}$ из модели в остаточных разностях появляется смещенный на ИС контур $6^{+}$с совпадающим знаком наклона производной в центре, отвечающим поглощению неона-20. График $3+$ это опыт с изотопом ${ }^{20} \mathrm{Ne}$, где содержание ${ }^{22} \mathrm{Ne}$ минимально. При удалении его контура из модели обработки в разностях появляется смещенный контур $3^{+}$с противоположным знаком наклона, отвечающим усилению неона- $22^{4}$.

Помимо этого в крыльях контуров буферных изотопов наблюдаются широкие неидентифицированные структу-

\footnotetext{
${ }^{4}$ По результатам численной обработки для амплитуд этих двух контуров получены значения $+3.46 \%$ и $-2.05 \%$.
}

ры (овалы), природу которых еще предстоит выяснять, а также узкие структуры с противоположным знаком $\left(6^{+}\right.$, вблизи $-600 \mathrm{Oе})$, возможно, демонстрирующие перенос с уровней $1 s_{2,4}$ вверх интегрального скрытого выстраивания, обнаруженного в [3].

\section{Обсуждение механизма наблюдаемой инверсии поглощения}

Как известно, причиной инверсии коэффициента поглощения на переходе $3 s_{2}-2 p_{4}$ могут быть или безызлучательные столкновительные переходы, или оптическая накачка. Столкновения второго рода - известный механизм безызлучательной передачи энергии возбуждения между атомами разного сорта $a$ и $b$, при которых энергия может передаваться от возбужденного атома $a^{*}$ к невозбужденному $b$, а возможный дефект энергии $\Delta E$ компенсируется за счет кинетической энергии относительного движения:

$$
a^{*}+b \rightarrow a+b^{*}+\Delta E .
$$

В частности, механизм создания инверсной населенности, обусловленный соударениями второго рода атомов неона в основном состоянии с возбужденными атомами гелия, находящимися в метастабильных состояниях $2{ }^{3} S_{1}$ и $2{ }^{1} S_{0}$ с дефектом энергии $\Delta E=313-1247 \mathrm{~cm}^{-1}$ $(-0.154-+0.215) \mathrm{eV}$, реализован в гелий-неоновых лазерах $[18,19]$. Специфика ситуации в том, что стационарный процесс преимущественного переноса энергии за счет ударов второго рода в одном направлении происходит только тогда, когда относительная заселенность возбужденных атомов, по крайней мере, одного сорта, поддерживается неравновесной [18], и можно пренебречь обратными переходами, что и приводит к инверсии населенности.

Если имеется смесь изотопов как в данном опыте, то в силу равновесного распределения атомов по энергетическим уровням в смеси изотопов обратные переходы надо учитывать. Однако поскольку безызлучательный обмен возбуждением есть результат столкновения двух изотопических атомов, у которых для идентичных уровней дефект энергии уровней $\Delta E$ отсутствует, то сечения прямого и обратного обмена возбуждением для них равны. Поэтому и после столкновения они останутся в тех же состояниях, что и до столкновения. Таким образом, в смеси изотопов безызлучательные столкновения не могут приводить к изменению населенностей уровней атомных переходов.

Поэтому источником инверсии может быть только резонансный механизм передачи энергии возбуждения между атомами разного сорта, возникающий при $\Delta E \sim 0$ в случае, когда частоты переходов перекрываются в пределах допплеровских ширин линий, и разрешены радиационные переходы атомов в основные состояния. Тогда возбужденные атомы, концентрация и интенсивность излучения которых больше, могут инициировать 


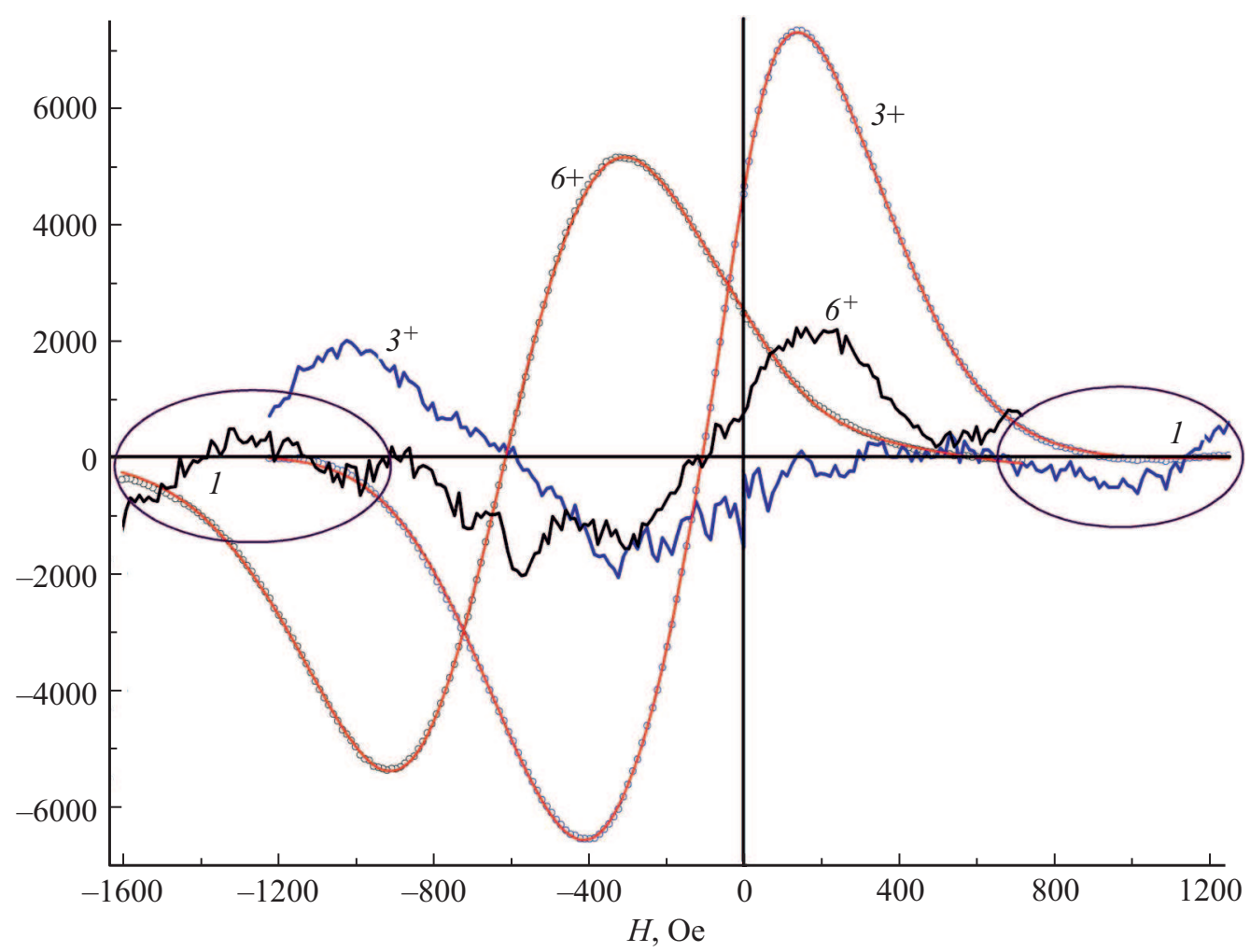

Рис. 5. Визуализация не включенных в модель контуров (остатков) по результатам численной аппроксимации опытов с малым содержанием примеси (неон-20, 3+) и с большим содержанием примеси (неон-22, 6+). 1 - неизвестная структура в крыльях, $3^{+}-$исключенный из подгонки контур примеси неона-22, $6^{+}-$исключенный из подгонки контур примеси неона-20. Масштаб остатков $1,3^{+}$и $6^{+}$увеличен в 10 раз.

вынужденные переходы „вверх“ из основного состояния в атомах другого сорта, реализуя процесс оптической накачки. Поскольку линии поглощения у газов весьма узки, то попасть в резонанс непросто. Тем не менее, такие совпадения имеют место. Так, в справочнике [19] описан случай перекрытия спонтанного излучения УФ линии гелия с резонансной линией цезия, приводящий к возбуждению уровней цезия и инверсии населенностей уровней цезия (генерации) на переходах в более низкие возбужденные состояния, которые возбуждаются из основного состояния только электронными столкновениями и в итоге оказываются менее заселенными.

В смеси изотопов одного элемента проблема совпадений частот в значительной мере отпадает. Изотопические сдвиг линий часто оказываются меньше их допплеровских ширин, и речь идет об исследовании конкретных случаев. В работах $[20,21]$ сообщалось также и об оптической самонакачке однокомпонентных газов со сверхтонкой структурой основного состояния. В плане исследования возможности инверсии в смеси изотопов следует сравнивать оптическую накачку верхних уровней исследуемых переходов с процессами возбуждения нижних уровней. Сначала рассмотрим в рамках упрощенной модели уровней принципиальную возможность образования инверсии в смеси изотопов с равновесными заселенностями уровней. Для чего построим и проанали-

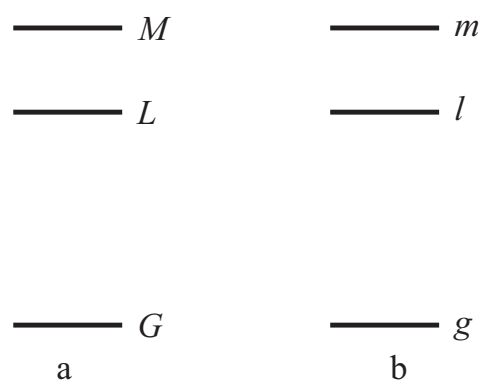

Рис. 6. Упрощенная схема уровней изотопов.

зируем балансные уравнения для заселенностей. А затем перейдем к анализу конкретной ситуации в изотопах неона.

Рассмотрим модель газа, содержащего два изотопа ( $a$ и $b$ ) и соответственно три пары уровней с одинаковыми энергиями (рис. 6). Для упрощения описания уровни изотопа большей концентрации будем обозначать заглавными буквами $M, L, G$, а второго (примесного) прописными $m, l, g$. При этом с уровней $M, m$ радиационные переходы в основные состояния $G$ и $g$ разрешены, a с уровней $L, l$ запрещены, но разрешены в другие нижележащие возбужденные состояния. Стационарный приход населенности, исходно обусловленный прямым 
электронным возбуждением из основного состояния на уровни $M, m$, описывается членами

$$
\langle Q e\rangle N e N_{G},\langle Q e\rangle N e N_{g},
$$

где $\mathrm{Ne}$ - концентрации электронов, $N_{G, g}-$ концентрация атомов в основном состоянии, Qe - сечение возбуждения уровней.

Для упрощения включим в эти члены и другие стационарные процессы, действующие в отсутствие обмена населенностью между изотопами: столкновительный и радиационный приход населенности вследствие возбуждения с более низких возбужденных уровней и приход населенности сверху вследствие спонтанного распада более высоких возбужденных уровней. Примем во внимание, что при равенстве вероятностей распада одноименных уровней, потенциалов ионизации изотопов и параметров разряда в отсутствие процессов обмена населенностью, распределение заселенностей по уровням изотопов равновесное, пропорциональное отношению концентраций изотопов в основных состояниях $\left(N_{m} / N_{M}=N_{g} / N_{G}\right)$. А возможность радиационного прихода населенности с основных состояний $N_{G}$ и $N_{g}$ „вверх“ на уровни $M, m$, которое индуцируется полем СИЗ партнера с уровней $M$ и $m$ в свое основное состояние, учтем членами

$$
\left(v_{G M}\right) N_{g},\left(v_{g m}\right) N_{G},
$$

где $v_{i j}$ - вероятности вынужденных переходов с основного состояния, инициируемые полями СИЗ изотопов, зависящими от населенности соответствующих уровней как

$$
v_{G M}=d_{\mathrm{ef}} \hbar \omega_{M G} A_{M G} N_{M}, \quad v_{g m}=d_{\mathrm{ef}} \hbar \omega_{m g} A_{m g} N_{m} .
$$

Здесь $d_{\mathrm{ef}}-$ множитель, учитывающий фактор формы (вытянутую геометрию разряда) и „время накопления“ излучения в разряде [22]. В силу равенства $A_{M G}=A_{m g}$ будем использовать для первых коэффициентов Эйнштейна обозначение $A$. Примем также $\hbar \omega_{M G}=\hbar \omega_{m g}=\hbar \omega$. С точностью до множителя населенности $N_{M}$ и $N_{m}$ допускают замену на $k N_{G}$ и $k N_{g}$, соразмеряющую относительный вклад стационарных процессов. Вынужденный полем СИЗ распад уровней $M, m$ принимать во внимание не будем. Он мал сравнительно со спонтанным распадом, который опишем членами

$$
N_{M} / \tau_{M}, N_{m} / \tau_{m},
$$

где $\tau_{M}$ и $\tau_{m}$ - времена жизни, обусловленные распадом на все нижележащие уровни. Поскольку нам важен принципиальный вопрос - будет ли иметь место передача энергии возбуждения между по-разному заселенными одноименными уровнями $M$ и $m$ изотопов, то примем времена жизни уровней одинаковыми и равными $\tau$.

На первый взгляд кажется естественным упрощением в плане реализации оптической накачки связывать приход населенности с основных состояний на уровни $M$ и $m$ с действием суммарного поля СИЗ с этих уровней. Однако сложение полей СИЗ с равными весами означает, что мы приравниваем самонакачку (самонасыщение) перехода полем его собственного СИЗ и оптическую накачку полем СИЗ партнерского перехода. Но это невозможно сделать, в частности, при ИС, превышающем ширину линии (убирающем оптическую накачку полем СИЗ партнера), а также при отсутствии ИС (превращающего смесь „изотопов“ в чистый газ, в котором инверсии также быть не должно). Анализ подобных балансных уравнений приводит к невозможности инверсии.

Поэтому для упрощения балансных уравнений и устранения парадоксов мы разделили члены, ответственные за оптическую накачку партнером, и члены, ответственные за самонасыщение. Самонасыщение было добавлено к перечисленным выше в (9) стационарным процессам, определяющим населенность уровней изотопов в отсутствие обмена (еще одним переопределением $\langle Q e\rangle N e)$ и множителя $k$. В наших опытах при изменении концентраций изотопов сумма концентраций изотопов удерживалась постоянной, т.е. выполнялось условие $N_{G}+N_{g}=1$. В итоге получаем балансные уравнения:

$$
\begin{gathered}
\left(\langle Q e\rangle N e+d_{\mathrm{ef}} \hbar \omega A k N_{g}\right) \tau=\frac{N_{M}}{1-N_{g}}, \\
\left(\langle Q e\rangle N e+d_{\mathrm{ef}} \hbar \omega A k\left(1-N_{g}\right)\right) \tau=\frac{N_{m}}{N_{g}} .
\end{gathered}
$$

Здесь вторые члены в скобках описывают приход населенности, индуцированный накачкой излучением партнерского перехода, которое определено через концентрацию атомов в его основном состоянии. Видно, что при равенстве концентраций изотопов в основном состоянии оказываются равными и концентрации изотопов на уровнях $M$ и $m$. При уменьшении концентрации примесного изотопа $N_{g}$ уменьшается член прихода в левой части уравнения (13) и, как следствие, падает населенность уровня $M$ относительно $N_{G}$. В уравнении (14) ситуация обратная: населенность уровня $m$ растет относительно $N_{g}$ при его уменьшении. Таким образом, уравнения (13),(14) демонстрируют перенос населенности между уровнями, связанными с основным состоянием.

Что касается нижнего уровня $l$, то в отсутствие механизма радиационного заселения с основного состояния его населенность при уменьшении $N_{g}$ уменьшается, и поэтому населенность уровня $m$ может превысить населенность уровня $l$, и коэффициент излучения на примесном переходе $m-l$ может изменить знак. Таким образом, накачка смеси изотопов их собственным излучением в принципе допускает образование инверсии переходов при уменьшении концентрации примесного изотопа.

Более детально рассмотрим возможности оптической накачки изотопов неона. В неоне с основным состоянием связаны уровни $n s_{2,4}, n d_{2,5}$ и $n s_{1}$, у которых $J=1$. Это УФ переходы с допплеровской шириной 
$k v \sim 10000 \mathrm{MHz}$, на порядок величины большей, чем в видимой области спектра, и с ИС от нескольких сот $\mathrm{MHz}$ для уровней $1 s$, до $3300 \mathrm{MHz}$ для высоких уровней. Здесь ИС всегда меньше допплеровских ширин и нет препятствий для радиационного обмена населенностью между одинаковыми уровнями изотопов с ростом населенности уровней примеси вследствие оптической накачки излучением буферного изотопа. На схеме рис. 2 отображены уровни $3 s_{2}, 2 p_{4}$ и $1 s_{2,4}$.

Нам интересен переход $3 s_{2}-{ }^{1} S_{0}$ (процесс 1 рис. 2 ), и поскольку переход с нижнего уровня $2 p_{4}$ исследуемого перехода в ${ }^{1} S_{0}$ в нашем случае запрещен, то дополнительный прирост населенности уровня $2 p_{4}$, обусловленный оптической накачкой, отсутствует, и инверсия населенности перехода $3 s_{2}-2 p_{4}$ кажется возможной, если, уменьшая концентрацию примести, уменьшить ниже определенного предела населенность уровня $2 p_{4}$. Однако здесь присутствует другой радиационный механизм прироста населенности уровня: „эффект самонасыщения“, обусловленный полем спонтанного распада с $2 p_{4}$ на расположенные ниже уровни, способный инициировать перенос заселенности вверх и подымать порог инверсии.

Сравним эффективность возбуждения уровней $3 s_{2}$ и $2 p_{4}$ вследствие оптической накачки (процесс 1 , рис. 2 ) и самонасыщения (процессы 2,3, рис. 2). Для начала рассмотрим вклад в населенность уровня $3 s_{2}$ самонасыщения (процесс 2, рис. 2) сравнительно с оптической накачкой уровня $3 s_{2}$ с основного состояния. Радиационный вклад в населенность состояний примесного изотопа, вынужденный излучением буферного изотопа, зависит от трех параметров - интенсивности вынуждающего поля (пропорционального частоте излучения, вероятности излучающего перехода и населенности его верхнего уровня), населенности уровня примесного изотопа, с которого осуществляется возбуждение, и степени перекрытия смещенных линий изотопов. Если сравнивать вклады различных процессов в возбуждение уровней перехода $3 s_{2}-2 p_{4}$, то при одинаковой населенности излучающего состояния $3 s_{2}$ видимое излучение отличается от УФ излучения длиной волны и вероятностью перехода.

Для УФ перехода (процесс 1, рис. 2) это величины $60 \mathrm{~nm} \mathrm{и} 2.63 \cdot 10^{7} \mathrm{~s}^{-1}$, для видимого (процесс 2, рис. 2) это $633 \mathrm{~nm}$ и $3.33 \cdot 10^{6} \mathrm{~s}^{-1}$, поэтому интенсивность УФ излучения, формируемая более энергетичными квантами, на 2 порядка величины больше, чем у линий видимого диапазона. В отношении населенности нижних уровней $1 s_{i}$ и $2 p_{j}$ известно, что первые заселены на 3-4 порядка величины меньше концентрации атомов в основном состоянии, а уровни $2 p_{i}$ заселены на 2-3 порядка меньше, чем уровни $1 s_{i}$. Таким образом, оптическая накачка уровня $3 s_{2}$ из основного состояния как минимум на 5 порядков величины эффективнее, чем полем СИЗ, поэтому вопрос о степени перекрытия линий изотопов на переходе $3 s_{2}-2 p_{4}$ уже не имеет значения ${ }^{5}$. Отметим, что для уровня $3 s_{2}$ оба процесса (накачка и самонасыщение) однонаправлены и способствуют приросту населенности и созданию инверсии на переходе $3 s_{2}-2 p_{4}$. Причем добавка самонасыщения к населенности уровня $3 s_{2}$ обеспечивается кроме уровня $2 p_{4}$ также восемью другими $2 p$-уровнями.

Сделаем сравнительную оценку изменения населенности уровней $3 s_{2}$ и $2 p_{4}$ примесного изотопа при насыщении полем СИЗ видимых линий буферного изотопа на переходах $2 p_{4}-1 s_{j}$ (процессы 3, рис. 2) и на переходах $3 s_{2}-2 p_{j}$. Здесь все длины волн одного порядка величины, близкие к длине волны перехода $3 s_{2}-2 p_{4}$. Вероятности переходов $2 p_{4}-1 s_{j}$ несколько больше $\left(1.4-2.6 \cdot 10^{7} \mathrm{~s}^{-1}\right)$, чем на переходах $3 s_{2}-2 p_{j}$, но число последних в 3 раза больше, и можно говорить о паритете прироста населенности самонасыщением, обусловленного вероятностями переходов. Однако одиндва порядка набегают за счет большей населенности уровня $2 p_{4}$ сравнительно с уровнем $3 s_{2}$. Таким образом, по интенсивности насыщающего поля излучения выигрыш $2 p_{4}$ сравнительно с $3 s_{2}$ составляет $1-2$ порядка. Существенно больший выигрыш набегает из-за большей населенности уровней $1 s$ сравнительно с уровнями $2 p$, и оптическое возбуждение уровня $2 p_{4}$ с уровней $1 s$ выглядит способным обеспечить прирост населенности, сравнимый с тем, который обеспечивает самонасыщение уровня $3 s_{2}$ полем СИЗ на уровни $2 p$ примесного изотопа. Поэтому вопрос о степени перекрытия данных линий изотопов становится значимым. И поскольку на этих переходах ИС более чем вдвое превышают их допплеровские ширины, то это почти на два порядка величины уменьшает эффективность оптического возбуждения с уровней $1 s$ (насыщение перехода). В результате сохраняются приоритетные условия для инверсии коэффициента поглощения на переходе $3 s_{2}-2 p_{4}$ примесного изотопа за счет оптической накачки уровня $3 s_{2}$ с состояния ${ }^{1} S_{0}$.

\section{Заключение}

Демонстрируемые в настоящей работе возможности роста относительной населенности уровней примесного изотопа неона вследствие оптической накачки переходов в основное состояние подтверждают высказанное во Введении предположение, что доложенный в [11] выход на плато ОМРПЧ, расположенного в окрестности 1350 Ое и порождаемого схожим с переходом $3 s_{2}-{ }^{1} S_{0}$ резонансным переходом $2 s_{4}-{ }^{1} S_{0}$ [23], объясняется именно таким процессом.

Существенный вклад могут давать и радиационные механизмы заселения $2 p$-уровней с нижележащих состо-

\footnotetext{
${ }^{5}$ Регистрация магнитооптических спектров на переходе $3 s_{2}-2 p_{4}$ дает информацию о населенностях уровней, сформированных процессами оптической накачки, которые с излучением самого этого перехода мало связаны. Поэтому даже лучше, если его изотопическое расщепление больше ширины линий и магнитооптические спектры изотопов не перекрываются, и плохо, если ИС мал. Тогда разрешать спектры изотопов и сравнивать поглощение становится сложнее.
} 
яний. Хотя в наших опытах с поглощением лазерного излучения $[10,12]$ и в данной работе были исследованы характеристики уровня $2 p_{4}$, полученные результаты могут быть распространены и на другие $2 p$-уровни. Все они также могут дополнительно заселяться радиационными переходами с нижележащих состояний, причем с преимущественным ростом населенности в центральной части разрядной трубки. Вследствие этого схожим образом будет расти и населенность более высоких уровней, заселяемых ступенчатыми переходами с уровней $2 p$. То, что ОМРПЧ с большей эффективностью порождаются в центре разрядной трубки, находящейся в однородной области магнитного поля соленоида, позволяет более точно определять их ИС и происхождение.

\section{Финансирование работы}

Работа выполнена в рамках приоритетных исследований СО РАН (Программа II.10.2.) и президентской программы поддержки ведущих научных школ (НШ-6896.2016.2).

\section{Благодарности}

Автор благодарит В.А. Сорокина за вклад в создание данной автоматизированной установки и В.А. Томилина за помощь в измерениях.

\section{Конфликт интересов}

Автор заявляет, что у него нет конфликта интересов.

\section{Список литературы}

[1] Чайка М.П. Интерференция вырожденных состояний. Л.: Изд-во ЛГУ, 1975.

[2] Александров Е.Б., Хвостенко Г.И., Чайка М.П. Интерференция атомных состояний. М.: Наука, 1991.

[3] Сапрыкин Э.Г., Селезнев С.Н., Сорокин В.А. // Письма в ЖЭТФ. 2002. Т. 76. С. 322.

[4] Раутиан С.Г., Сапрыкин Э.Г. // Опт. и спектр. 2002. Т. 92. № 2. C. 385.

[5] Им Тхек-де, Сапрыкин Э.Г., Шалагин А.М. // Опт. и спектр. 1973. Т. 35. В. 2. С. 202.

[6] Раутиан С.Г. // Письма в ЖЭТФ. 1994. Т. 60. С. 462.

[7] Раутиан С.Г. // ЖЭТФ. 1995. Т. 108. С. 1186.

[8] Барыбин И.В., Сорокин В.А., Чурин А.Е. // Опт. и спектр. 2003. T. 95. № 4. C. 929.

[9] Сапрыкин Э.Г., Селезнев С.Н., Сорокин В.А. // ЖЭТФ. 2003. T. 124. C. 1232.

[10] Сапрыкин Э.Г. // Опт. и спектр. 2017. Т. 123. № 2. С. 285.

[11] Сапрыкин Э.Г. // ЖЭТФ. 2016. Т. 149. С. 233.

[12] Сапрыкин Э.Г. // Опт. и спектр. 2017. Т. 122. № 4. С. 568.

[13] Сапрыкин Э.Г., Сорокин В.А. // Опт. и спектр. 2010. Т. 109. № 4. C. 573.

[14] Лисиџын В.Н., Чеботаев В.П. // ЖЭТФ. 1968. Т. 54. С. 419.

[15] Им Тхек-де, Казаниев А.П., Раутиан С.Г., Сапрыкин Э.Г., Шалагин А.М. // Квант. электрон. 1974. Т. 1. № 2. С. 416.

[16] Казанцев А.П. // Опт. и спектр. 1974. Т. 37. № 6. С. 1028.
[17] Eikema K.S.E., Ubachs W., Hogervorst W. // Phys. Rev. A. 1994. V. 49. P. 803-808.

[18] Бочкова О.П., Фриш С.Э. // Спектроскопия газоразрядной плазмы. Л.: Наука, 1970. С. 319.

[19] Справочник по лазерам / Под ред. А.М. Прохорова. Т. 1. М.: Советское радио, 1978.

[20] Александров Е.Б., Якобсон Н.Н. // Письма ЖЭТФ. 1977. T. 26. C. 463.

[21] Александров Е Б., Якобсон Н.Н. // Опт. и спектр. 1980. T. 48. B. 4. C. 828.

[22] Вайнштейн Л.А., Мироненко В.Р., Раутиан С.Г., Сапрыкин Э.Г. // Опт. и спектр. 1999. Т. 87. В. 3. С. 372.

[23] Сапрыкин Э.Г. // Опт. и спектр. 2016. Т. 120. № 2. С. 222. 